\title{
Willingness to Wait for a Vaccine Against COVID-19: Results of a Preference Survey
}

\author{
Tommi Tervonen $^{1,2}$ - Aura Cecilia Jimenez-Moreno ${ }^{1,3} \cdot$ Nicolas Krucien $^{1} \cdot$ Heather Gelhorn ${ }^{4} \cdot$ Kevin Marsh $^{1}$. \\ Sebastian Heidenreich ${ }^{1}$
}

Accepted: 13 November 2020 / Published online: 14 December 2020

(c) Springer Nature Switzerland AG 2020

\section{Dear Editor,}

The coronavirus disease 2019 (COVID-19) pandemic has caused a significant loss of life and developed into a historic threat to numerous healthcare systems around the world [1-5]. In response, many governments have imposed public health interventions with the aim of slowing down the spread of the SARS-Cov-2 virus causing COVID-19 [6-8]. While varying between countries, these interventions often included self-isolation, social distancing, closure of public places, restricted internal movement, and stay-at-home requirements [9]. Despite being effective in slowing the spread of the disease, the policies have detrimental effects on the economy, elective health care provision, mental health, and societal freedom $[10,11]$.

To pave a way for an exit strategy from imposed public health interventions, the development of medical treatments and vaccines has become a priority for governments worldwide [12]. However, while various drugs are being developed or repurposed to treat COVID-19 [13-17], the possibility remains that burdensome and costly interventions, such as social distancing, can only be lifted after a vaccine is available. Currently, there are over 100 vaccines in development [18], but even with the "pandemic speed"

Electronic supplementary material The online version of this article (https://doi.org/10.1007/s40271-020-00483-y) contains supplementary material, which is available to authorized users.

Tommi Tervonen

tommi.tervonen@evidera.com

Patient-Centered Research, Evidera, London, UK

2 Department of Epidemiology, University of Groningen, University Medical Center Groningen, Groningen, The Netherlands

3 Wellcome Trust Research Centre, Newcastle University, Newcastle-upon-Tyne, UK

4 Patient-Centered Research, Evidera, Bethesda, MD, USA at which pharmaceutical development is proceeding [19], it was expected to take 12-18 months for a vaccine to become available for the general public [20]. Furthermore, with the COVID-19 cases falling in several countries and many vaccines as well as treatments being tested in parallel, clinical trials may not be able to demonstrate efficacy with the same statistical power usually required for regulatory approval $[12,21]$. In this context, registrational trials of COVID-19 vaccines are expected to be considered successful if they achieve protection for $50 \%$ of the vaccinated individuals for 6 months, which may not be sufficient to create a population-level immunity that warrants lifting of burdensome public health interventions [22]. Hence, societies may face a trade-off between vaccine efficacy and the duration of implemented interventions such as social distancing.

We used an online survey to elicit participants' willingness to wait for a surely working vaccine (i.e., a vaccine that will work in everyone vaccinated). The first part of the survey contained a thresholding exercise where participants answered choice tasks with two options: to either wait for 6 months for a vaccine that works only in 50\% of the people vaccinated, or to wait longer for a surely working vaccine (Fig. 1). The participants were told that there are enough resources to develop only one of these two vaccines. Each participant answered two to four such choice tasks, where the time until surely working vaccine becomes available was varied. The thresholding exercise captured each participant's willingness to wait for a surely working vaccine in ranges of $<9$ months, $9-12$ months, $12-15$ months, $15-18$ months, 18-21 months, or $>21$ months. In addition to the preference elicitation tasks, the survey included a dominance test where the waiting time for both vaccine options was 6 months. This was used to assess whether participants meaningfully processed the presented information, by calculating the share of people who choose the surely working vaccine with the same waiting time as the alternative, less efficacious vaccine. The survey also included a brief socio-demographic 


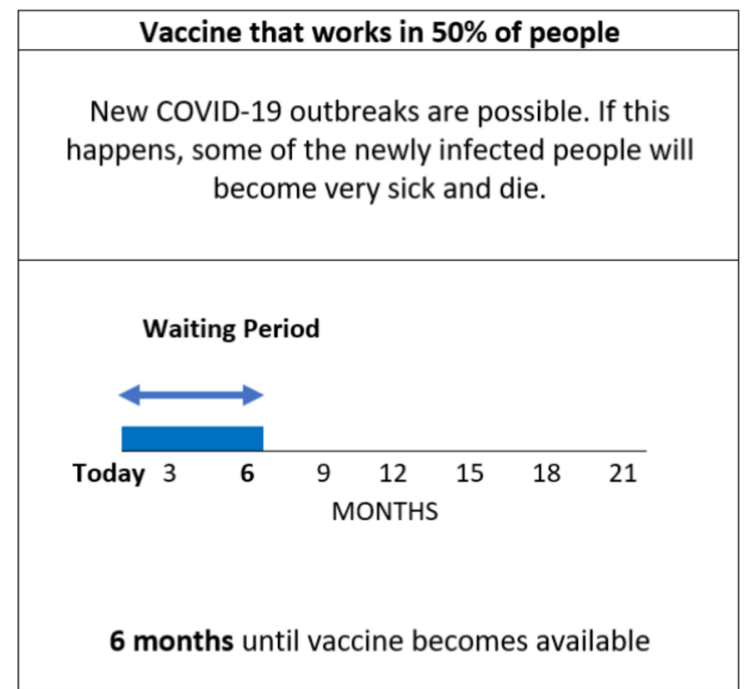

Fig. 1 Example choice task

questionnaire and an open-ended question on participants' opinions on social distancing measures to be implemented by their governments. The complete survey is included in the Electronic Supplementary Material.

The survey was pre-tested with preference researchers who are not part of the research team. We fielded the survey from 5 May 2020 to 29 May 2020 by disseminating the survey link through social media posts in Twitter and LinkedIn. The survey also encouraged the participants to share the link to their social networks. No financial incentives were offered for completing the survey and there were no exclusion criteria for participating.

A total of 1964 participants started and 1184 completed the survey. Of these, 1069 (90\%) answered the dominant choice task correctly and were included in the analysis with no missing data (Supplementary Fig. 1). Participants were predominantly female $(n=737 ; 69 \%)$, between 18 and 44 years of age $(n=723 ; 67 \%)$, residents of Europe $(n=644 ; 60 \%)$, and employed $(n=650 ; 61 \%)$ (Supplementary Table 1). Of those who were employed at the time of the survey, $31 \%(n=335)$ expressed that their work had been affected by the COVID-19 pandemic and 15 (1\%) of the $22(2 \%)$ who were unemployed (seeking work or unable to work) at the time of the survey attributed their unemployment to the COVID-19 impacts. Over 20\% $(n=229)$ of the participants reported belonging to a group at higher risk from COVID-19 with a "lung condition that is not severe" being the most common reason $(n=62)$. Twenty-four $(2 \%)$ participants reported belonging to the very high-risk group.

Fifty-nine percent $(n=630)$ of survey participants were willing to wait at least 18 months for a surely working vaccine and more than half of survey participants $(n=545$; $51 \%$ ) were willing to wait for longer than the maximum of

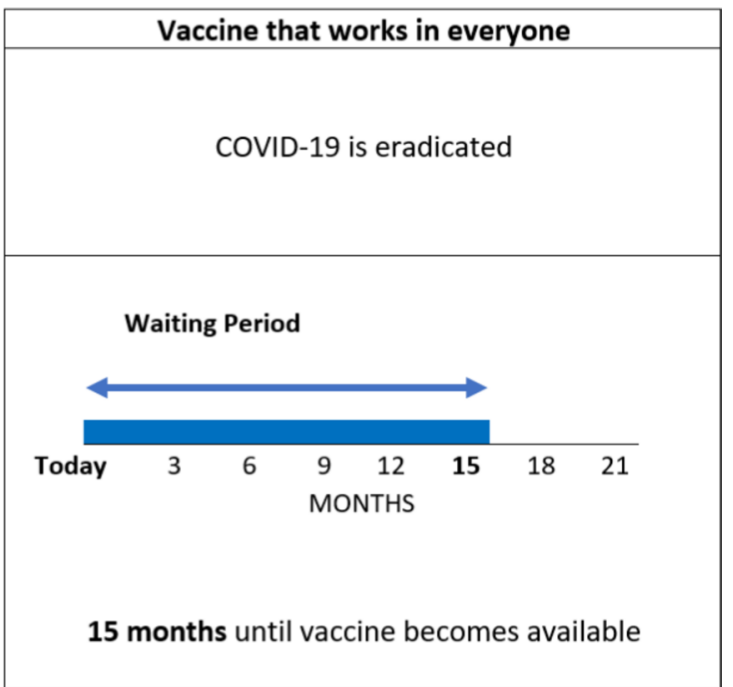

21 months included in the survey (Fig. 2). One hundred and seven $(10 \%)$ participants were willing to wait $<9$ months. Participant characteristics had a significant effect on willingness to wait (Supplementary Table 2), with males willing to wait less time than females (OR 0.59, 95\% CI 0.46-0.75) and participants in the COVID-19 risk group willing to wait longer (OR 1.69, 95\% CI 0.69-2.27). When controlling for age, gender, economic status, and COVID-19 risk group, participants resident in countries outside of Europe, USA, and Canada were willing to wait less time than participants in Europe (OR 0.72, 95\% CI 0.52-0.99).

Most participants $(n=790 ; 67 \%)$ answered the open-text question about the social distancing measure they think their governments should impose (Supplementary Table 3). Out of these responses, 69 (9\%) belonged to participants failing the dominance test. A total of 978 entries were qualitatively analyzed by adding a descriptive code. The most endorsed measures referred to restrictions on individuals $(n=504$; $52 \%$ of all coded entries) with minimum physical distance as the most common restriction ( $n=195$ out of 504 entries) followed by measures on an individual's hygiene and personal protective equipment ( $n=188 ; 19 \%)$, where use of masks were most commonly mentioned ( $n=154$ out of 188 entries). The second most common category was measures affecting the community ( $n=324 ; 32 \%$ of all coded entries), with restrictions on public mass gatherings being the most endorsed measure ( $n=90$ out of 324 entries). Restrictions directly affecting work or businesses were the least mentioned $(n=150 ; 15 \%)$ and in this category, working from home was the most common measure $(n=61$ out of 150 entries).

The results of this study suggest that among those responding to the survey, more than half would be willing 
Lower risk overall $(n=840)$

Higer risk overall $(n=229)$
Lower risk female $(n=584)$

Higher risk female $(n=153)$
Lower risk male $(n=256)$

Higher risk male $(n=76)$
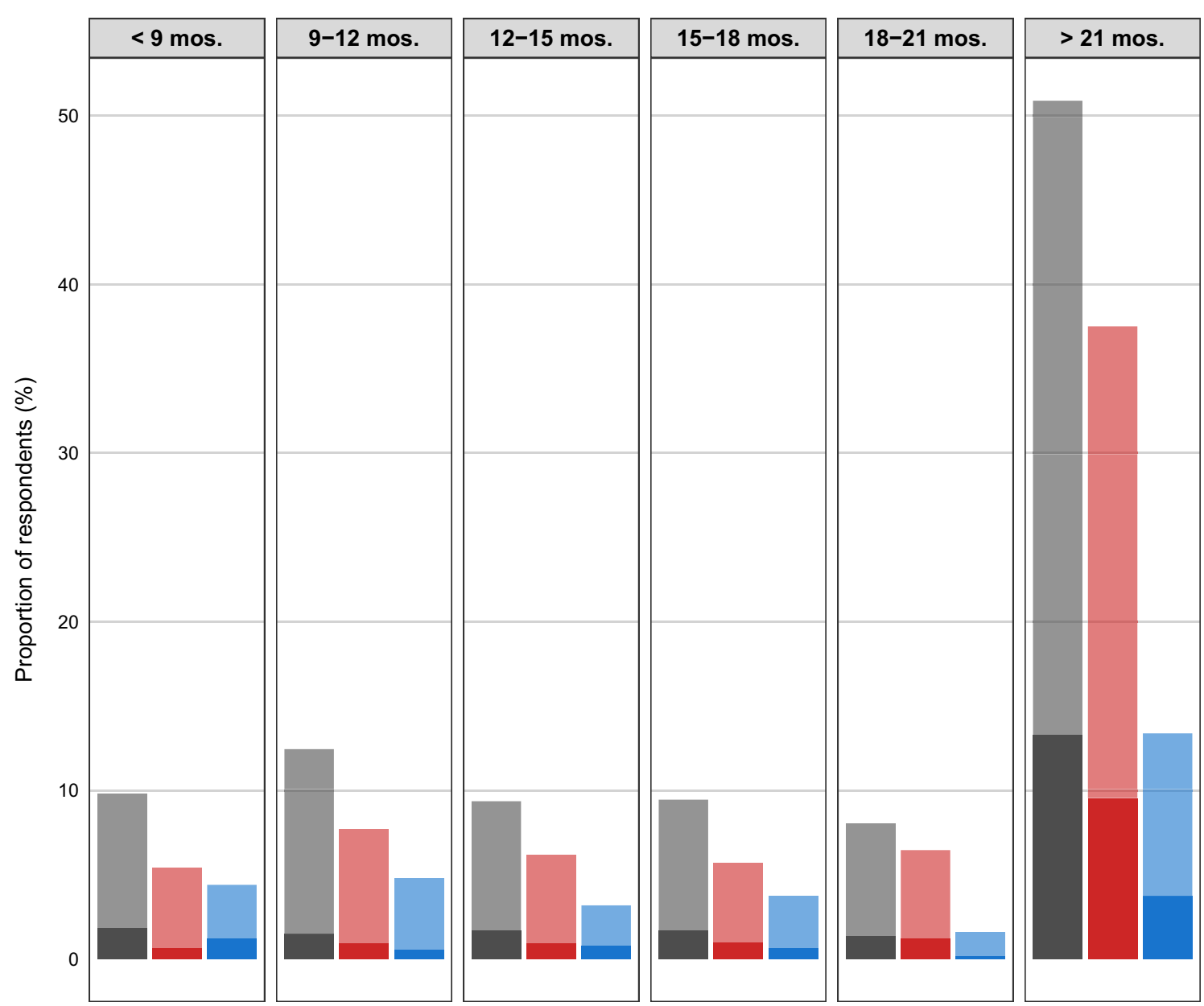

Fig. 2 Willingness to wait in months (mos.) for a surely working vaccine instead of waiting 6 months for a vaccine that works in 50\% of people. Higher risk group consists of participants who Public Health England considers to be at high or very high risk from COVID-19

to endure ongoing social distancing measures for at least an additional 12 months if that resulted in an effective vaccine against COVID-19. Participants from higher risk groups were willing to wait significantly longer for an effective vaccine than participants from the lower risk group, reflecting the lower benefit-tolerability ratio that a potentially ineffective vaccine would represent to them. The results also suggest that women are willing to wait significantly longer for a vaccine than men, despite having a lower risk of suffering from severe COVID-19 symptoms. Only a small share of participants wanted a vaccine as fast as possible, if that meant the vaccine had lower efficacy.

This study has various limitations. The participants in the study were recruited using convenience sampling through social media and were overwhelmingly young and female; therefore, the sample is not representative of the general public in most countries. The survey did not include the possibility that an effective vaccine might never be developed, and not mentioning this explicitly may have led participants to indicate that they are willing to wait longer than they otherwise may have. The choice options were mutually exclusive even though in practice a less efficacious vaccine could be developed first, followed by a more efficacious vaccine. Participants were unable to denote that they prefer public health interventions to be lifted before a vaccine has been developed. Finally, the preference elicitation exercise deliberately simplified the vaccine choice context to average efficacy and waiting time, excluding other potentially relevant attributes such as risk of side effects.

The survey was fielded in May 2020 and at this time, social distancing measures had been in place for approximately 7 weeks in many of the affected countries [24-27]. It is not clear to what extent participants' willingness to wait may change over time after prolonged experience with social 
distancing. Furthermore, many respondents at lower risk from COVID-19 were willing to wait very long for a vaccine even though the societal distancing measures are likely to have a large impact on their lives. Different governments enforced different measures at the time of this cross-sectional survey, and we did not ask participants which social distancing measures they followed. The existence of perceived societal cost or moral obligations should be addressed in future research.

Despite the limitations of this study, our findings clearly suggest that governments and regulators should carefully consider the level of evidence required for vaccines to be granted marketing authorization, even if this prolongs clinical trial programs. Fast approvals of vaccines that result in suboptimal immunization of the general public may risk losing the public's trust in policy decisions [23].

Acknowledgements The authors would like to thank Caitlin Thomas (Evidera) for survey validation and Emily Sargent (Evidera) for editorial support. We also thank all study participants and those who shared the survey link.

\section{Declarations}

Funding This study has received no external funding.

Conflict of interest The authors have no conflicts of interest relevant for this study.

Data sharing All study data is available from the corresponding author (TT) on request.

Guarantor statement TT takes responsibility for the content of the manuscript, including the data and analysis.

Author contributions TT participated in conception, design, planning and conduct of the study, analysis and interpretation of data. CJM participated in design, planning and conduct of the study, and in analysis and interpretation of data. SH and $\mathrm{HG}$ participated in conception and design of the study and interpretation of data. NK participated in analysis and interpretation of data. KM participated in conception and design of the study.

\section{References}

1. Bartsch SM, Ferguson MC, McKinnell JA, et al. The potential health care costs and resource use associated with COVID-19 in the United States: a simulation estimate of the direct medical costs and health care resource use associated with COVID-19 infections in the United States. Health Aff. 2020;39(6):927-35.

2. Giannakeas V, Bhatia D, Warkentin MT, Bogoch II, Stall NM. Estimating the maximum capacity of COVID-19 cases manageable per day given a health care system's constrained resources. Ann Intern Med. 2020;173(5):407-10.

3. Grasselli G, Pesenti A, Cecconi M. Critical care utilization for the COVID-19 outbreak in Lombardy, Italy: early experience and forecast during an emergency response. JAMA. 2020;323(16):1545-6.
4. Shoukat A, Wells CR, Langley JM, Singer BH, Galvani AP. Projecting demand for critical care beds during COVID-19 outbreaks in Canada. CMAJ. 2020;192(19):E489-96.

5. Sohrabi C, Alsafi Z, O'Neill N, et al. World Health Organization declares global emergency: a review of the 2019 novel coronavirus (COVID-19). Int J Surg. 2020;76:71-6.

6. Anderson RM, Heesterbeek H, Klinkenberg D, Hollingsworth TD. How will country-based mitigation measures influence the course of the COVID-19 epidemic? Lancet. 2020;395(10228):931-4.

7. Koo JR, Cook AR, Park M, et al. Interventions to mitigate early spread of SARS-CoV-2 in Singapore: a modelling study. Lancet Infect Dis. 2020;20(6):678-88.

8. Hellewell J, Abbott S, Gimma A, et al. Feasibility of controlling COVID-19 outbreaks by isolation of cases and contacts. Lancet Glob Health. 2020;8(4):e488-96.

9. Imai N, Gaythorpe KAM, Abbott S, et al. Adoption and impact of non-pharmaceutical interventions for COVID-19. Wellcome Open Res. 2020;5:59.

10. Courtemanche C, Garuccio J, Le A, Pinkston J, Yelowitz A. Strong social distancing measures in the United States reduced the COVID-19 growth rate. Health Aff. 2020;39(7):1237-46.

11. Pan A, Liu L, Wang C, et al. Association of public health interventions with the epidemiology of the COVID-19 outbreak in Wuhan. China. JAMA. 2020;323(19):1915-23.

12. COVID-19 Clinical Research Coalition. Global coalition to accelerate COVID-19 clinical research in resource-limited settings. Lancet 2020; 395(10233):1322-5.

13. Abrams EM, Szefler SJ. COVID-19 and the impact of social determinants of health. Lancet Respir Med. 2020;323(19):1915-23.

14. Armitage R, Nellums LB. COVID-19 and the consequences of isolating the elderly. Lancet Public Health. 2020;5(5):e256.

15. Organisation for Economic Co-operation and Development. Evaluating the initial impact of covid-19 containment measures on economic activity', OECD Technical Report. 2020. https:// read.oecd-ilibrary.org/view/?ref=126_126496-evgsi2gmqj\&title =Evaluating_the_initial_impact_of_COVID-19_containmen t_measures_on_economic_activity. Accessed 19 June 2020.

16. Sanders JM, Monogue ML, Jodlowski TZ, Cutrell JB. Pharmacologic treatments for coronavirus disease 2019 (COVID-19): a review. JAMA. 2020;323(18):1824-36.

17. Ueda M, Martins R, Hendrie PC, et al. Managing cancer care during the COVID-19 pandemic: agility and collaboration toward a common goal. J Natl Compr Canc Netw. 2020;18(4):1-4.

18. Le Thanh T, Andreadakis Z, Kumar A, et al. The COVID19 vaccine development landscape. Nat Rev Drug Discov. 2020;19(5):305-6.

19. Lurie N, Saville M, Hatchett R, Halton J. Developing covid-19 vaccines at pandemic speed. N Engl J Med. 2020;382(21):1969-73.

20. Lancet The. COVID-19: fighting panic with information. Lancet. 2020;395(10224):537.

21. Hotez PJ, Corry DB, Bottazzi ME. COVID-19 vaccine design: the Janus face of immune enhancement. Nat Rev Immunol. 2020;20(6):347-8.

22. GlobalData Healthcare. Covid-19 vaccines may only need to reach lowest efficacy bar for approval. 19 May 2020. https://www.clini caltrialsarena.com/comment/covid-19-vaccine-efficacy-approval/. Accessed 19 June 2020.

23. Trogen B, Oshinsky D, Caplan A. Adverse consequences of rushing a SARS-CoV-2 vaccine: implications for public trust. JAMA. 2020;323(24):2460-1.

24. Agencia EFE. Brasil se prepara para el inicio de las restricciones ante el avance del COVID-19. 15 March 2020. https://www.efe. com/efe/america/sociedad/brasil-se-prepara-para-el-inicio-delas-restricciones-ante-avance-del-covid-19/20000013-4196413. Accessed 19 June 2020. 
25. Dunford D, Dale B, Stylianou N, Lowther E, Ahmed M, de la Torre Arenas I. Coronavirus: The world in lockdown in maps and charts. 7 April 2020. https://www.bbc.com/news/world-52103747. Accessed 19 June 2020.

26. Hirsch C. Europe's coronavirus lockdown measures compared. 15 April 202031 March 2020. https://www.politico.eu/article/europ es-coronavirus-lockdown-measures-compared/. Accessed 19 June 2020.

27. Statista.com. Number of school/district closures due to the coronavirus (COVID-19) in the United States as of March 17, 2020, by status. 18 June 2020. https://www.statista.com/statistics/11030 64/covid-19-school-closures-status-us/. Accessed 19 June 2020. 